\title{
Fourier transform spectrometer developed for high repetition rate mid- infrared supercontinuum sources
}

\author{
A. Khodabakhsh, M. Nematollahi, K. E. Jahromi, R. Krebbers, M. A. Abbas, F. J. M. Harren \\ Trace Gas Research Group, Department of Molecular and Laser Physics, Institute for Molecules and Materials, \\ Radboud University, 6525 AJ Nijmegen, The Netherlands
}

Fourier transform spectroscopy is a well stablished method, with a unique feature of providing broad spectral coverage and high spectral resolution. Incoherent thermal light sources, which are omnidirectional and provide a low spectral brightness, are traditionally used in combination with this method. Therefore, a long averaging time is needed to obtain a high signal-to-noise ratio (SNR) spectrum and the detection sensitivity is usually low for gas phase samples. Newly developed ultra-broadband, low-noise, mid-infrared supercontinuum (SC) light sources show a great potential to replace the thermal sources in Fourier transform spectrometers (FTSs). They are spatially coherent and can deliver orders of magnitude higher spectral brightness compared to thermal sources, while providing equal or even higher spectral bandwidth [1]. The main drawback of SC sources, historically, has been their high relative intensity noise (RIN) due to noise amplification in the nonlinear broadening process [2]. Recent advancements in diode-laser-pumped mid-infrared SC sources provide reduced RIN by utilizing higher repetition rates and shorter pulse durations [3]. The high repetition rate of these sources can also be utilized in combination with a synchronous demodulation technique to reduce the $1 / \mathrm{f}$ noise in the detection system. Here we present a home-built FTS, using only out-of-shelf optical components, especially developed to work with ultra-broadband and high repetition rate mid-infrared SC sources. The FTS can potentially work in the 2-11 $\mu \mathrm{m}$ bandwidth with a minimum spectral resolution of $1 \mathrm{GHz}$ suitable for detection of gases at atmospheric pressure. To increase the light-matter interaction length, we employed a multipass cell with $32 \mathrm{~m}$ optical path length. Figure 1 demonstrates the performance of the system in combination with the SC source, in three different cases of (a) using a single photodetector operating in the baseband, (b) synchronous demodulation of the photodetector output referenced to the SC repetition rate and (c) utilizing a balanced detection scheme in the baseband. In all of the experiments, the gas sample consists of a mixture of $5 \mathrm{ppm}$ Ethane and $25 \mathrm{ppm}$ Ethyl acetate, diluted in $\mathrm{N}_{2}$ at atmospheric pressure.
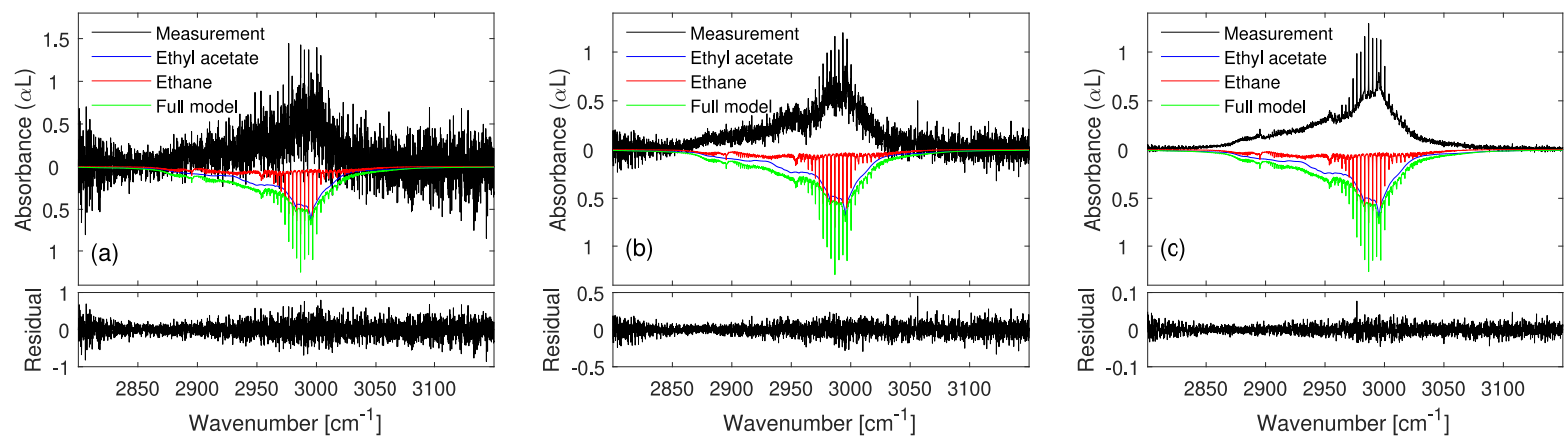

Fig. 1 The measured (with $3 \mathrm{GHz}$ spectral resolution and 15 minutes averaging) and fit model spectra using three different detection method with the FTS (a) a single detector in the baseband, (b) a single detector followed by a lock-in amplifier referenced to the repetition rate of the SC source, and (c) balanced detection in the baseband. The fit model spectra are inverted for clarity.

As expected, using a single detector in the baseband, Fig.1 (a), yields a low SNR due to the RIN of the SC source. Using a lock-in amplifier for synchronous detection at the repetition frequency of the SC source, Fig.1 (b), reduces the $1 / \mathrm{f}$ noise and provides a higher SNR in the detected spectrum. However, baseband balanced detection, Fig.1 (c), can provide the highest improvement in the SNR of the spectrum. The lock-in amplifier approach only maps the intensity noise of the source at its repetition frequency to the spectrum. Therefore, although it yields better results compared to the baseband detection, the pulse to pulse fluctuations of the SC source are directly translated into the noise in the spectrum. In the balanced detection scheme the common RIN noise of the laser on the two detectors are effectively subtracted and cancelled (its efficiency depends on the intensity and phase matching of the two detected interferograms), yielding the best results.

\section{References}

[1] C. R. Petersen, P. M. Moselund, L. Huot, L. Hooper, and O. Bang, "Towards a table-top synchrotron based on supercontinuum generation," Infrared Phys. Technol. 91, 182-186 (2018).

[2] N. R. Newbury, B. R. Washburn, K. L. Corwin, and R. S. Windeler, "Noise amplification during supercontinuum generation in microstructure fiber," Opt. Lett. 28, 944-946 (2003).

[3] K. Kwarkye, M. Jensen, R. D. Engelsholm, M. K. Dasa, D. Jain, P. Bowen, P. M. Moselund, C. R. Petersen, and O. Bang, "In-amplifier and cascaded mid-infrared supercontinuum sources with low noise through gain-induced soliton spectral alignment," Scientific Reports 10 , 8230 (2020). 